\title{
Efectos fisiológicos agudos de un dispositivo para ejercitarse en casa Acute physiological response to exercising with a piece of home equipment
}

Johnny Alberto Montoya Arroyo, Jimena Ramírez Cambronero, Luis Fernando Aragón Vargas

Universidad de Costa Rica (Costa Rica)

\begin{abstract}
Resumen. A la luz de la importancia de la actividad física regular como un elemento clave en la prevención de muchas enfermedades, se reportan a continuación los resultados del estudio realizado con un aparato para ejercitarse en la casa. 22 estudiantes universitarios jóvenes, aparentemente saludables, participaron en el estudio. Se midió el efecto agudo de 10 minutos de ejercicio con el dispositivo sobre la frecuencia cardíaca, el costo energético y la circulación periférica en las piernas. Se contrastan los resultados obtenidos con las afirmaciones que se hacen en distintas versiones de publicidad del aparato en cuestión. No se obtuvieron efectos fisiológicos agudos significativos de la utilización del dispositivo durante 10 minutos continuos: la frecuencia cardíaca aumentó apenas 8 latidos por minuto con respecto al reposo y la temperatura cutánea no varió o más bien disminuyó. El costo energético no fue significativamente mayor mientras se usaba el dispositivo en comparación con la condición de reposo. Estos resultados sugieren que aquellas personas que utilicen este dispositivo en sustitución de la actividad física regular estarán sacrificando beneficios importantes para su salud.
\end{abstract}

Palabras clave: gasto energético, máquina de ejercicio, hogar, actividad física.

Abstract. The importance of doing regular physical activity (exercise) as a key element in the prevention of several diseases is well known. The following experiment was performed to evaluate the acute physiological effects of a home-exercise machine on 22 young, apparently healthy, university students. While the individuals exercised in the machine for ten (10) minutes, measures of heart rate, energy expenditure and peripheral circulation to the legs were taken and recorded. The results were contrasted against the claims made in different ads promoting the selected apparatus. There were no significant acute physiological effects resulting from the utilization of the machine during a 10-minute period. Heart rate increased only 8 beats per minute (bpm) from baseline and the temperature measured on the legs showed no variation or was even reduced. Energy expenditure was not significantly higher while using the device in comparison with the resting condition. These results suggest that consumers using this home-exercise equipment as a substitute of performing regular physical activity will be sacrificing important health benefits.

Keywords: energy expenditure, exercise machine, home, physical activity.

\section{Introducción}

Actualmente existe un claro consenso entre los científicos del movimiento humano y las autoridades de salud pública, tanto a nivel nacional como internacional, sobre los efectos nocivos de la inactividad física y el sedentarismo (OMS, 2010). El problema es serio pues, por ejemplo, se estima que en Costa Rica alrededor de $50 \%$ de la población es físicamente inactiva (Ministerio de Salud \& Ministerio de Deporte y Recreación, Costa Rica, 2011), a pesar de los esfuerzos de distintas entidades relacionadas con la salud pública.

Mientras tanto, la población está siendo bombardeada por publicidad engañosa que la induce, directa o indirectamente, a no asumir un estilo de vida físicamente activo. Junto a unas pocas ofertas que son sólidas y

Fecha recepción: 05-06-20. Fecha de aceptación: 27-07-21

Johnny Alberto Montoya Arroyo

jhonny.montoyaarroyo@ucr.ac.cr confiables, se anuncian máquinas, suplementos y accesorios que prometen maravillosos resultados, en muy poco tiempo y con muy poco esfuerzo. Estas promesas rara vez se cumplen, lo cual podría causar un serio perjuicio sobre la salud de la población, que se ve defraudada y renuncia a la actividad física regular.

El conocimiento acumulado y los métodos de evaluación desarrollados por los profesionales en ciencias del movimiento humano permiten evaluar objetivamente las afirmaciones publicitarias que se hacen sobre distintas máquinas, suplementos y accesorios para moldear el cuerpo o perder peso (Swain, 2009). Dichas evaluaciones serían equivalentes a las que se realizan en farmacia para evaluar la efectividad de distintos medicamentos.

La evaluación de cualquier dispositivo para ejercitarse en la casa puede centrarse en los efectos que tiene su utilización regular (efectos crónicos o adaptaciones) (Wilmore et al., 1985), o en los efectos agudos obtenidos (respuestas fisiológicas inmediatas) (Montoya, 
Ramírez y Aragón, 2021). El estudio de los efectos crónicos es más atractivo, pues permite verificar si las personas realmente pierden peso, disminuyen su porcentaje de grasa corporal, o mejoran la masa muscular, la fuerza o el consumo máximo de oxígeno, entre otras variables de interés. Sin embargo, ética y científicamente no se justifica realizar una investigación experimental si no hay buenas razones para suponer que el tratamiento bajo estudio podría ser beneficioso. Antes de investigar los efectos crónicos, por lo tanto, tiene sentido estudiar detenidamente los efectos agudos, especialmente si la utilidad aparente de un dispositivo es cuestionable. La actividad física moderada, con la cual se compara el equipo en cuestión en la publicidad, tiene efectos agudos y crónicos ampliamente documentados. La caminata a paso moderado $\left(4,8 \mathrm{~km}^{*} \mathrm{~h}^{-1}\right)$, por ejemplo, representa una intensidad de 3,5 MET o un gasto energético de $174 \mathrm{kcal}$ en una hora para una persona de $50 \mathrm{~kg}$ de peso $(246 \mathrm{kcal}$ en una hora para alguien de 70 $\mathrm{kg}$ ) (Ainsworth et al., 2011, código 21045). Esa velocidad de caminata eleva la frecuencia cardíaca a 95-130 latidos por minuto, dependiendo de la condición física de la persona (American Heart Association, s.f.).

El dispositivo bajo estudio, vendido comercialmente como Legxercise ${ }^{\circledR}$ (Modelo LEGX-011, Legxercise, China; distribuido por Intellibrands LLC, Miami, FL, EE.UU.) es un aparato sencillo, con dos plataformas rectangulares para que el usuario coloque los pies mientras está cómodamente sentado. Un motor eléctrico mueve las plataformas horizontalmente, haciendo que los pies se muevan hacia adelante y atrás (desplazamiento total $=58 \mathrm{~mm}$ ); la amplitud del movimiento articular es de $7^{\circ}$ en las rodillas y de $10^{\circ}$ en los tobillos. La cadencia del movimiento es de 24-25 ciclos por minuto en la velocidad baja y 26,5 a 27,5 ciclos por minuto en la velocidad alta (la frecuencia varía ligeramente según el peso de las piernas de la persona). Entre las afirmaciones que se hacen en la publicidad se presenta el movimiento pasivo de los pies como un ejercicio cardiovascular eficaz y saludable que promueve una circulación sanguínea adecuada, «mejora la circulación sin necesidad de un ejercicio agotador», «convierte estar sentado en un ejercicio eficaz y saludable», «ayuda a que la sangre corra hasta el corazón» (Legxercise Tevecompras, 2016).

Por lo tanto, el propósito de esta investigación fue poner a prueba el LegXercise $\mathbb{R}$ en un grupo de adultos jóvenes para verificar si el aparato era capaz de producir efectos similares a los que se obtienen haciendo ejercicio moderado tipo caminata, como lo proclama la publicidad. Concretamente, se midió el efecto agudo so- bre la frecuencia cardíaca, el costo energético y la circulación periférica en las piernas, durante 10 minutos de ejercicio con el dispositivo (a saber, el tiempo sugerido de uso en el manual de usuario).

\section{Metodología}

Se contó para el estudio con una población de 22 estudiantes, 11 mujeres y 11 hombres, con una edad de $19,5 \pm 1,4$ años, un peso de $63,7 \pm 10,4 \mathrm{Kg}$ y una estatura de $166,6 \pm 7,3 \mathrm{~cm}$, todos aparentemente saludables; se obtuvo consentimiento informado conforme al procedimiento aprobado por el Comité Ético Científico Institucional de la Universidad de Costa Rica, documento VI-8797-2017, en el cual se respetan todos los requisitos de la Declaración de Helsinki. Así mismo la población participante se presentó al laboratorio una única vez para completar todas las mediciones.

\section{Procedimientos}

La población participante se presentó al laboratorio, en donde primeramente se registró la masa corporal (MC) de cada participante hasta los 50 gramos más cercanos y su estatura hasta el milímetro más cercano, utilizando un estadiómetro (Seca ${ }^{\circledR}$, modelo 2861500009 , Hamburgo, Alemania). El siguiente paso fue estimar su porcentaje de grasa corporal mediante bioimpedancia (Seca ${ }^{\circledR}$ modelo 514, Hamburgo, Alemania). Según BosyWestphal, et al. (2013), la validez de la medición con ese equipo de la masa libre de grasa y por ende de su contraparte, la masa grasa, en un modelo de composición corporal de dos compartimentos es sumamente alta, para un coeficiente de determinación $\mathrm{R}^{2}=0.98$; el mismo estudio reporta un valor de confiabilidad para una muestra de 15 participantes con mediciones duplicadas: el error técnico de medición fue de $0,221 \mathrm{~kg}$ de masa grasa, error que corresponde a 1,27\% de la media de medición. Así mismo a cada participante se le colocó un monitor de frecuencia cardíaca (FC) Polar ${ }^{\circledR}$, modelo FT7 (Kempele, Finlandia) el cual tiene una precisión de la medición de $\pm 1 \%$ o $\pm 1 \mathrm{ppm}$ (Polar FT7 Manual del Usuario). Además, se le colocó el equipo para medir el consumo de oxígeno, que se registró con un carro metabólico Jeager MasterScreen CPX (CareFusion Corporation, San Diego, California) (CARMET), con el fin de obtener el gasto calórico de forma indirecta. Finalmente, se midió la temperatura cutánea del tren inferior por medio de una cámara Termográfica (Flir, modelo: 650sc, Wilsonville-Oregón) la cual cuenta con una precisión de la medición aproximadamente de $\pm 1 \%$ 
en un objetivo entre $5^{\circ} \mathrm{C}$ y $120^{\circ} \mathrm{C}$ (Flir T650SC).

A cada participante se le realizó una medición y registro de $\mathrm{VO}_{2}$ según CARMET, en estado de reposo en posición decúbito supino durante 10 minutos. Luego se colocó sentado en una silla frente a la máquina para ejercicio LegXercise colocando los pies sin zapatos sobre el dispositivo, conforme a las instrucciones del fabricante. El aparato se encendió y se ajustó a la velocidad máxima (26.5 ciclos de movimiento por minuto); cada participante lo utilizó durante 10 minutos de nuevo, conforme a las instrucciones del fabricante. El gasto energético aproximado según $\mathrm{VO}_{2}$ se registró durante el tiempo que utilizó el dispositivo al igual que en el estado de reposo, así como también se realizó la termografía del tren inferior durante el periodo que se utilizó la máquina (EJER).

Con el monitor de FC, se registró la FC promedio de los diez minutos tanto en el estado de reposo como durante el EJER. Del CARMET, se utilizó el consumo de oxígeno promedio obtenido en condición de reposo para cada participante, en $\mathrm{mL}^{*} \mathrm{~kg}^{-1} * \mathrm{~min}^{-1}$, como 1 MET; así mismo el valor obtenido en EJER se registró como múltiplos del consumo de oxígeno de cada participante en reposo o múltiplos del índice metabólico en reposo, MET (Physical Activity Guidelines Advisory Committee, 2008). Para obtener el gasto energético bruto en kilocalorías durante el ejercicio por calorimetría indirecta, se utilizó la siguiente fórmula:

Gasto energético bruto ${ }_{\text {CARMET }}=\left(\right.$ MET $\left._{\text {ejer }}\right) *(3.5 \mathrm{~mL}$ $\left.\mathrm{O}_{2} * \mathrm{~kg}^{-1} * \mathrm{~min}^{-1}\right) * \mathrm{MC} *\left(4.8 \mathrm{kcal} / 1000 \mathrm{~mL} \mathrm{O}{ }_{2}\right) * 10 \mathrm{~min}$ Donde:

$\mathrm{MET}_{\text {ejer }}$ es el múltiplo del equivalente metabólico o el índice metabólico en reposo obtenido para cada participante durante el ejercicio (como recomiendan Hills, Mokhtar y Byrne, 2014),

MC es la masa corporal de cada participante, y

10 min es el tiempo del esfuerzo con el dispositivo

El gasto energético neto se obtuvo con la misma fórmula, pero restando 1 MET a cada valor.

Para la termografía del tren inferior se registró en forma continua específicamente en las siguientes zonas anatómicas: vasto lateral (Z1, en la porción muscular más próxima a la inserción), tibial anterior (Z2, en la porción muscular más próxima al origen) y el gastrocnemio lateral (Z3, en la porción muscular más próxima al origen), todas las mediciones registradas en la pierna izquierda de cada participante. Del video, se obtuvo la temperatura cutánea en cada una de las tres zonas anatómicas mencionadas, en el minuto 0 (reposo), minuto 2:30, minuto 5 , minuto $7: 30$ y minuto 10 de ejercicio, todos \pm 2 segundos en el tiempo de medición, esto porque la medida se tomaba con un cursor predefinido y con la pierna siempre en la misma posición.

\section{Análisis estadístico}

Se calculó la estadística descriptiva (media \pm D.E.) de las características generales, para caracterizar la muestra. Además, se comparó a los hombres y las mujeres con pruebas t de Student. Para verificar la normalidad de los datos, se utilizó la prueba de KolmogorovSmirnov en cada una de las variables dependientes.

Para el análisis de los efectos fisiológicos, se realizaron ANOVA de dos vías de medidas repetidas (sexo X condición) con la frecuencia cardíaca y el gasto energético como variables dependientes. Además, con la temperatura superficial localizada como variable dependiente, se realizó un ANOVA de dos vías de medidas repetidas (sexo X tiempo de medición) para cada una de las tres zonas anatómicas, aplicando la corrección según Greenhouse-Geisser cuando la prueba de esfericidad de Mauchly dio significativa.

\section{Resultados:}

En la tabla 1 se desglosan las características básicas de la población participante en el estudio, divididos entre hombres y mujeres.

Tabla 1.

\begin{tabular}{|c|c|c|}
\hline & Mujeres $(\mathrm{n}=11)$ & Hombres $(\mathrm{n}=11)$ \\
\hline & Media \pm D.E. & Media \pm D. E. \\
\hline Edad (años cumplidos)* & $20,1 \pm 1,5$ & $19,0 \pm 1,0$ \\
\hline Estatura $(\mathrm{m})^{*}$ & $1,630 \pm 0,081$ & $1,702 \pm 0,040$ \\
\hline Peso (kg) & $61,01 \pm 10,81$ & $66,49 \pm 9,73$ \\
\hline Grasa corporal $(\%) *$ & $31,4 \pm 5,2$ & $17,7 \pm 6,3$ \\
\hline Frecuencia cardíaca en reposo $\left(\right.$ lat $\left._{*} \min ^{-1}\right)$ & $73,8 \pm 10,4$ & $75,8 \pm 11,0$ \\
\hline
\end{tabular}

En cuanto a los análisis inferenciales, de acuerdo con la prueba de normalidad de Kolmogorov-Smirnov, se obtiene que solamente en las variables de frecuencia cardíaca en reposo y gasto energético en reposo, mostraron una desviación de la normalidad ( $\mathrm{p}<0.001 \mathrm{y} p$ $=0.038$, respectivamente). En las figuras $1 \mathrm{y} 2$, se muestran los resultados de dichas variables dependientes.

La figura 1 muestra que la frecuencia cardíaca en reposo $\left(74,8 \pm 10,5\right.$ lat $\left.{ }_{*} \min ^{-1}\right)$ fue significativamente menor que en ejercicio $\left(82,9 \pm 10,4\right.$ lat $\left.{ }_{*} \min ^{-1}\right)(p=$ $0,016)$. No hubo diferencia entre hombres y mujeres $(p=0,855)$ ni tampoco hubo una interacción significativa entre condición y sexo $(\mathrm{p}=0,423)$.

En la figura 2 se muestra la comparación del gasto 


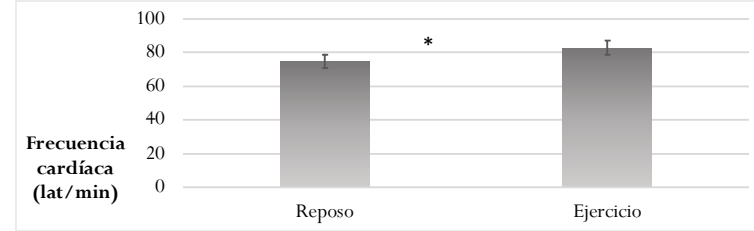

Figura 1. Comparación de la frecuencia cardíaca del grupo completo $(\mathrm{n}=22) .\left({ }^{*}\right) \mathrm{p}<0,01$

energético bruto en reposo $(44,11 \pm 9,28 \mathrm{~kJ})$ y en ejercicio $(46,38 \pm 8,06 \mathrm{~kJ})$ en diez minutos. La interacción entre sexo y condición fue significativa $(\mathrm{F}=13,11$, $\mathrm{p}=0,002)$, por lo cual se debe hacer un análisis de los efectos simples. Los hombres tuvieron un gasto energético significativamente más alto $(\mathrm{p}<0,05)$ que las mujeres en la condición de reposo: su promedio fue de 49,93kJ (IC95\% = 45,34 a 54,51kJ) en 10 minutos, vs. $38,30 \mathrm{~kJ}(\mathrm{IC} 95 \%=33,71 \mathrm{a} 42,88 \mathrm{~kJ})$ para las mujeres. Por otra parte, el aparente aumento en el gasto energético de las mujeres que se observa en la figura 2 no fue significativo para $\mathrm{p}<0,05$, ya que su media durante el ejercicio solo alcanzó a 45,39kJ (IC95\% $=40,22$ a $50,53 \mathrm{~kJ})$.

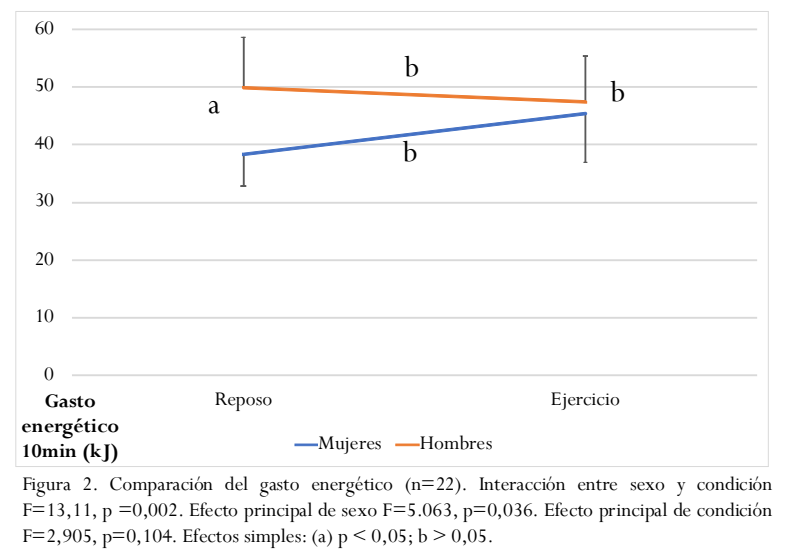

La figura 3 muestra la temperatura cutánea localizada para cada una de las tres zonas anatómicas a lo largo del tiempo, para las mujeres y los hombres. La interacción entre sexo y tiempo de medición no fue significativa para ninguna de las tres zonas $(\mathrm{p}>0,218)$. El efecto principal del tiempo de medición sí fue significativo para las zonas 2 y $3(\mathrm{p}<0,001)$, mostrando una disminución de la temperatura con respecto al tiempo inicial (en reposo). El efecto principal del sexo sí fue significativo, con promedios mayores para los varones en las tres zonas anatómicas: en el vasto lateral, 31,32 $\pm 1,16$ vs. $29,68 \pm 0,85^{\circ} \mathrm{C}(\mathrm{p}=0,001)$; en el tibial anterior, $31,68 \pm 0,83$ vs. $30,35 \pm 0,78^{\circ} \mathrm{C}(\mathrm{p}=0,001)$; en el gastrocnemio lateral, $31,18 \pm 1,35$ vs. $29,98 \pm$ $0,73^{\circ} \mathrm{C}(\mathrm{p}=0,015)$, para hombres vs. mujeres, respectivamente.
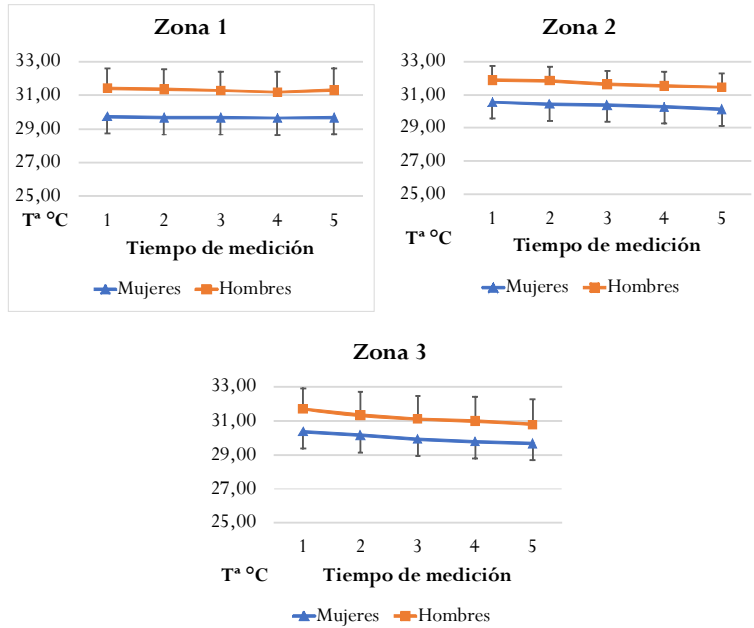

Figura 3. Comparación de la temperatura superficial localizada en las tres regiones, $\mathrm{n}=22$. Fig 3a, zona 1, vasto lateral: interacción $\mathrm{F}=0,706, \mathrm{p}=0,513$; tiempo de medición $\mathrm{F}=2,216, \mathrm{p}$ $=0,116$; sexo $\mathrm{F}=13,624, \mathrm{p}=0,001$. Fig 3b, zona 2, tibial anterior: interacción $\mathrm{F}=0,837$, $\mathrm{P}$ $=0,432$; tiempo de medición $\mathrm{F}=29,895, \mathrm{p}<0,001 ; \operatorname{sexo} \mathrm{F}=14,713, \mathrm{p}=0,001$. Fig 3c, zona 3, gastrocnemio lateral: interacción $\mathrm{F}=0,646, \mathrm{p}=0,498$; tiempo de medición $\mathrm{F}=$ $37,326, \mathrm{p}<0,001$; sexo $\mathrm{F}=7,020, \mathrm{p}=0,015$.

\section{Discusión}

El principal resultado de este estudio es que no se obtuvieron efectos fisiológicos agudos significativos de la utilización del LegXercise ${ }^{\circledR}$ durante 10 minutos continuos: la frecuencia cardíaca aumentó apenas 8 latidos por minuto con respecto al reposo, sin un aumento significativo en el gasto energético. La temperatura cutánea en las piernas no varió o más bien disminuyó, lo cual no apunta a un aumento en la circulación. A manera de referencia, el gasto bruto promedio obtenido usando la máquina durante diez minutos $(46,38 \mathrm{~kJ})$ representaría 278,3 kJ o 66,5 kilocalorías en una hora, el mismo gasto energético bruto que tendría una persona de $50 \mathrm{~kg}$ de peso quieta de pie, sentada, o descansando acostada sin hacer nada, según los códigos 07040, 07021, o 07011 de actividad física, respectivamente (Ainsworth et al., 2011). Esto difícilmente corresponde a «un ejercicio eficaz y saludable», como afirma la publicidad del equipo que además, al tener por nombre LegXercise, sugiere que es para hacer ejercicio.

Esta no es la primera vez que se documenta la exageración en que incurre la publicidad de algunos dispositivos para hacer ejercicio en el hogar. Un estudio reciente (Montoya Arroyo, et al., 2021) analizó el gasto energético real de un aparato de vibración mediante calorimetría indirecta por consumo de oxígeno, llegando a la conclusión de que el gasto energético fue de $1 /$ 50 del gasto declarado en la publicidad.

Por otro parte, la temperatura cutánea tuvo un comportamiento consistente en las tres zonas evaluadas (Figura 3): en una zona se mantuvo constante y cuando hubo variación estadísticamente significativa a lo largo 
del tiempo, esta fue una disminución, contrariamente a lo esperado como consecuencia del ejercicio. Las cámaras termográficas se han utilizado exitosamente para medir circulación periférica (Bagavathiappan et al., 2009; Carpes et al., 2018; Cholewka et al., 2017; Stathopoulos et al., 2015), pero es posible que la temperatura cutánea localizada no sea lo suficientemente sensible a variaciones agudas en la circulación periférica consecuentes a un ejercicio liviano. Para estar seguros de su sensibilidad, hicimos una prueba independiente en la cual un varón adulto realizó extensiones de rodilla en una máquina Cybex ${ }^{\circledR}$ modelo VR1-Legextension/Leg curl (Cybex International, Medway, MA, EE.UU.) con el mínimo peso disponible $(11,36 \mathrm{~kg})$ durante $10 \mathrm{minu}-$ tos continuos, pero con pausas de 1 segundo después de cada repetición. La temperatura en el vasto lateral fue de $30,9^{\circ} \mathrm{C}$ al iniciar, y aumentó a $31,4^{\circ} \mathrm{C}$ a los $2: 30 \mathrm{~min}$, $31,5^{\circ} \mathrm{C}$ a los $5: 00 \mathrm{~min}, 32^{\circ} \mathrm{C}$ a los $7: 30 \mathrm{~min}$ y $31,9^{\circ} \mathrm{C}$ a los 10 minutos de ejercicio.

En las tres zonas anatómicas evaluadas, las mujeres tuvieron temperaturas cutáneas aproximadamente $1^{\circ} \mathrm{C}$ más bajas que los hombres (Figura 3). La diferencia no solamente se presenta durante la utilización del equipo, sino también antes de iniciar (tiempo 0 minutos). Es posible suponer que eso se debe al efecto aislante de la grasa subcutánea que favorecería la conservación del calor corporal, ya que la temperatura ambiental en el laboratorio durante las mediciones fue de $26,4^{\circ} \mathrm{C} \pm$ $0,8^{\circ} \mathrm{C}$ (con una humedad relativa de $66 \%$, ligeramente por encima de la recomendada para obtener buenos registros) (Carpes et al., 2018). Esa temperatura ambiental estuvo claramente por debajo de la temperatura corporal normal. Las mujeres en nuestra muestra tuvieron un porcentaje de grasa significativamente mayor al de los hombres (Tabla 1), lo cual sugiere mayor grasa subcutánea.

A la luz de la ausencia de efectos agudos significativos y fisiológicamente relevantes, no pareciera existir justificación para realizar un experimento con el propósito de evaluar el efecto crónico de la utilización de este dispositivo para ejercicio en casa. Además, si bien en la publicidad se menciona que brinda una mejoría en la sensación de piernas cansadas, variable que no se contempló en el presente estudio, los resultados sugieren que, aun si este alivio de piernas fuera real y estadísticamente significativo, aquellas personas que utilicen este dispositivo en sustitución de la actividad física regular como el caminar, trotar o montar en bicicleta, estarían perdiendo una serie de importantes beneficios para su salud.

\section{Agradecimiento}

Se agradece a todas las personas participantes por su disposición a colaborar con este estudio, así como también al personal administrativo del CIMOHU, por su asistencia técnica y administrativa y, por último, al proyecto VI-838-B6-766 de la Universidad de Costa Rica por la colaboración en el financiamiento de esta investigación.

\section{Referencias}

Ainsworth, B. E., Haskell, W. L., Herrmann, S. D., Meckes, N., Bassett, D. R., Tudor-locke, C., Greer, J. L., Vezina, J., Whitt-glover, M. C., \& Leon, A. S. (2011). 2011 Compendium of Physical Activities: A Second Update of Codes and Met Values. Medicine \& Science in Sports \& Exercise, 43(8), 1575-1581. https://doi.org/10.1249/MSS.0b013e31821ece12 American Heart Association (s.f.). Target Heart Rates Chart. Recuperado de https: / /www.heart.org/en/ healthy-living/fitness / fitness-basics / target-heartrates

Asamblea Médica Mundial. (1964). Declaración de Helsinki: Principios éticos para las investigaciones médicas en seres humanos. Recuperado de http:// www.conamed.gob.mx/prof_salud/pdf/ helsinki.pdf

Bagavathiappan, S., Saravanan, T., Philip, J., Jayakumar, T., Raj, B., Karunanithi, R., Panicker, T. M. R., Korath, M. P., \& Jagadeesan, K. (2009). Infrared thermal imaging for detection of peripheral vascular disorders. Journal of Medical Physics / Association of Medical Physicists of India, 34(1), 43-47. https: / / doi.org/10.4103/0971-6203.48720

Bosy-Westphal1, A., Schautz1, B., Later1,W., Kehayias, JJ., D Gallagher, D., and Muller, MJ. (2013). European Journal of Clinical Nutrition. 67, S14-S21. https://doi:10.1038/ejcn.2012.160

Carpes, F. P., Mello-Carpes, P. B., Priego Quesada, J. I., Pérez-Soriano, P., Salvador Palmer, R., \& Cibrian Ortiz de Anda, R. M. (2018). Insights on the use of thermography in human physiology practical classes. Adv Physiol Educ, 42, 521-525. https://doi.org/ doi:10.1152/advan.00118.2018. Cholewka, A., Kajewska, J., Marek, K., Sieroñ-StoBtny, K., \& Stanek, A. (2017). How to use thermal imaging in venous insufficiency? Journal of Thermal Analysis and Calorimetry, 130(3), 1317-1326. https://doi.org/ 10.1007/s10973-017-6141-7 
Flyr T650sc. Descargado el 29 de mayo de 2021 de http: / / www.flirmedia.com / MMC/THG/ Brochures/RND_061/RND_061_US.pdf

Hills, A. P., Mokhtar, N., y Byrne, N. M. (2014). Assessment of physical activity and energy expenditure: an overview of objective measures. Frontiers in Nutrition 1(5),1-16. doi: https: / / doi.org/ 10.3389/fnut.2014.00005

Legxercise Tevecompras. (2016). [VideoYouTube]. Descargado el 24 de marzo de 2020 de https:// www.youtube.com/watch? $\mathrm{v}=\mathrm{ME} 49$ Aw0yosk

Ministerio de Salud, \& Ministerio de Deporte y Recreación, Costa Rica. (2011). Plan Nacional de Actividad Física y Salud 2011-2021. Descargado el 3 de marzo de 2020 de https: / / goo.gl/NEqhK6

Montoya Arroyo, J., Ramírez Cambronero, J., \& Aragón Vargas, L. (2021). Medición del gasto energético real por usar un producto comercial para ejercitarse en el hogar. Pensar En Movimiento: Revista De Ciencias Del EjercicioY La Salud, 19(1), e40646. https: / / doi.org/ 10.15517/pensarmov.v19i1.40646

OMS, O. M. de la S. (2010). Recomendaciones mundiales sobre actividad física para la salud. http: / / www.who.int/dietphysicalactivity/publications/ 9789241599979/es/

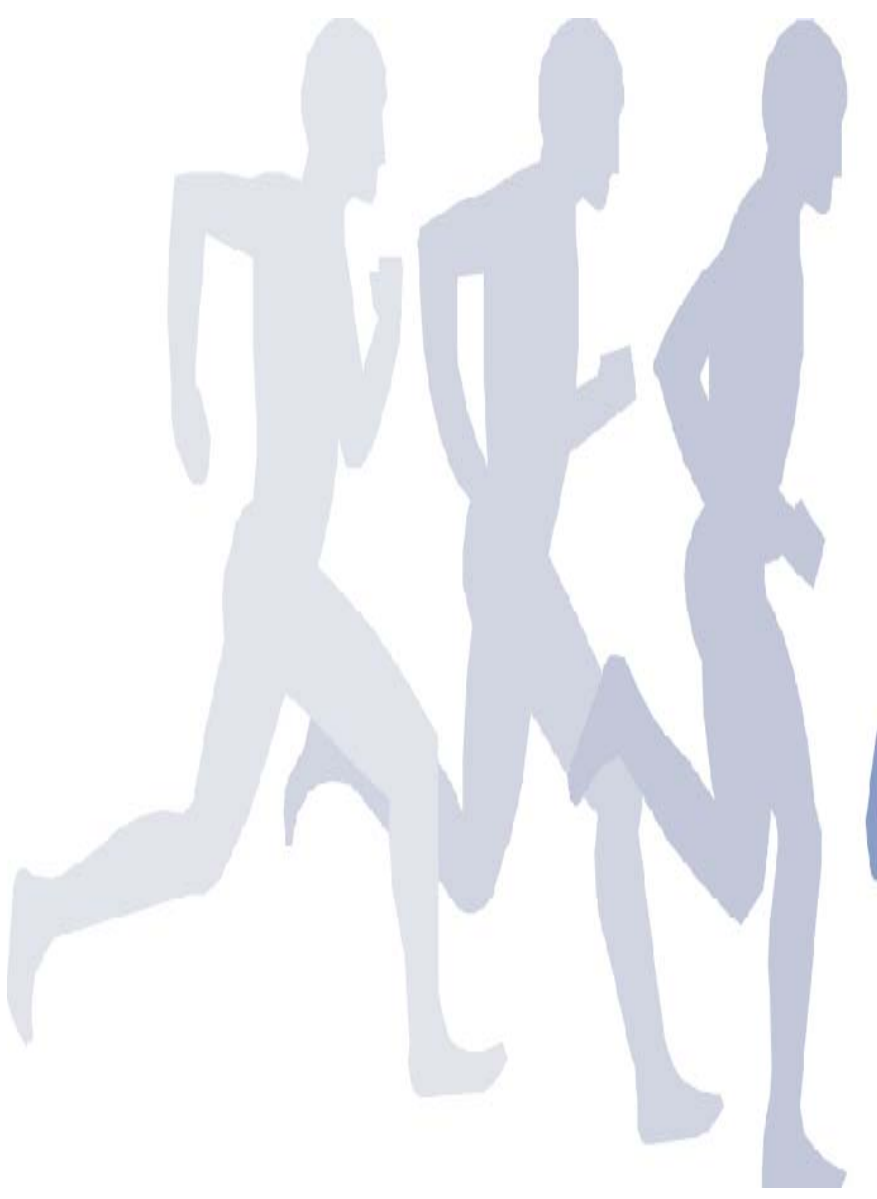

Physical Activity Guidelines Advisory Committee. (2008). Advisory Report | health.gov. Descargado el 3 de marzo de 2020 de https: / / health.gov/ourwork/physical-activity/previous-guidelines/2008physical-activity-guidelines/advisory-report

Polar FT7 manual de usuario. Descargado el 29 de mayo de 2021 de https: / / support.polar.com/ e_manuals/FT7/Polar_FT7_user_manual_Espanol/ ch06.html

Stathopoulos, I., Skouroliakou, K., Michail, C., \& Valais, I. (2015). Dynamic Infrared Thermography Study of Blood Flow Relative to Lower Limp Position. Journal of Physics Conference Series, 637(1), 012027. https: / /doi.org/10.1088/1742-6596/637/1/012027

Swain, D. P. (2009). EXERCISE EQUIPMENT:Assessing the Advertised Claims. ACSM's Health \& Fitness Journal, 13(5), 8-11. https://doi.org/10.1249/ FIT.0b013e3181b47bdc

Wilmore, J. H., Atwater, A. E., Maxwell, B. D., Wilmore, D. L., Constable, S. H., \& Buono, M. J. (1985). Alterations in Body Size and Composition Consequent to Astro-Trimmer and Slim-Skins Training Programs. Research Quarterly for Exercise and Sport, 56(1), 90-92. https://doi.org/10.1080/ 02701367.1985 .10608439

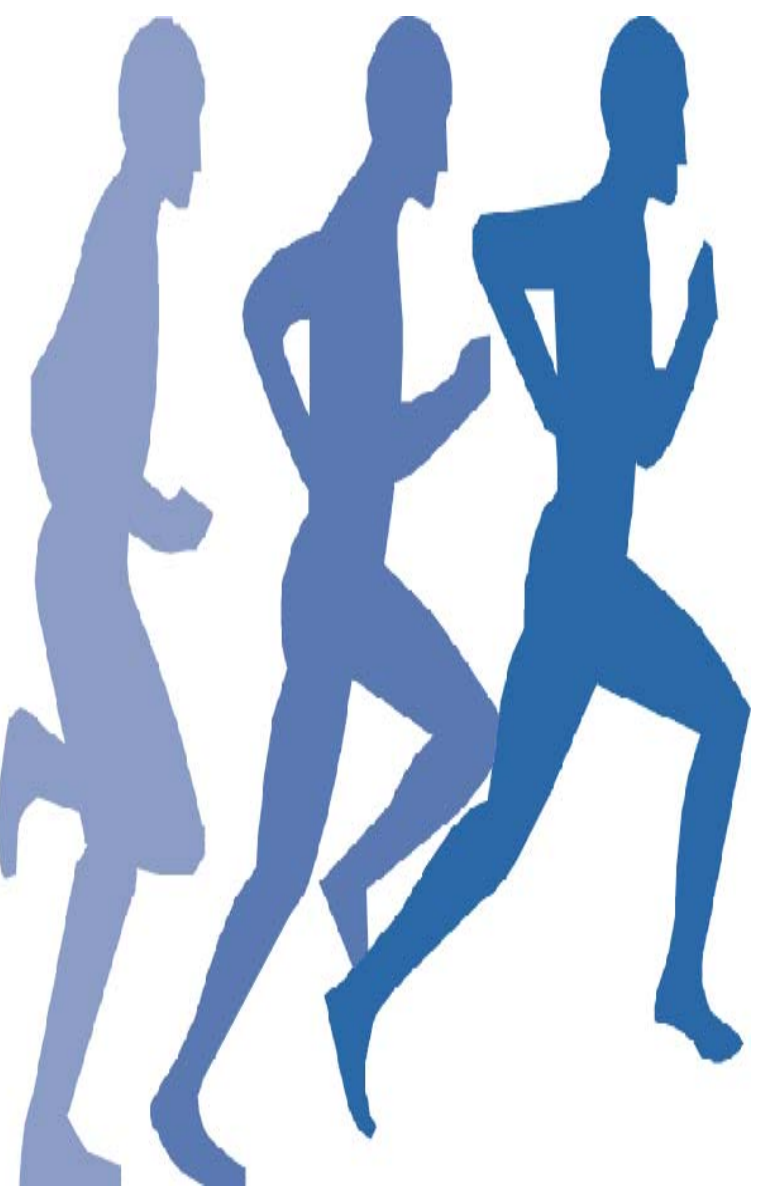

